\title{
Identifying the Support of Rectangular Signals in Gaussian Noise
}

\author{
Jiyao Kou \\ Department of Statistics, Stanford University
}

July 23, 2018

\begin{abstract}
We consider the problem of identifying the support of the block signal in a sequence when both the length and the location of the block signal are unknown. The multivariate version of this problem is also considered, in which we try to identify the support of the rectangular signal in the hyperrectangle. We allow the length of the block signal to grow polynomially with the length of the sequence, which greatly generalizes the previous results in 16. A statistical boundary above which the identification is possible is presented and an asymptotically optimal and computationally efficient procedure is proposed under Gaussian white noise in both the univariate and multivariate settings. The problem of block signal identification is shown to have the same statistical difficulty as the corresponding problem of detection in both the univariate and multivariate cases, in the sense that whenever we can detect the signal, we can identify the support of the signal. Some generalizations are also considered here: (1) We extend our theory to the case of multiple block signals. (2) We also discuss about the robust identification problem when the noise distribution is unspecified and the block signal identification problem under the exponential family setting.

Keywords: Block signal; Rectangular signal; Support identification; Multi-dimensional; Penalized Scan.
\end{abstract}

\section{Introduction}

Block signal detection and identification in a long one-dimensional sequence is a challenging and important problem and arises in many applications, for example, in epidemiology [13, 17] and Copy Number Variation [16, 21]. Block signal detection determines whether there exists any block signal in the sequence while block signal identification further identifies the support of the block signal. There has been a large body of work on signal detection, see, e.g. [15, 10, on the scan statistic; 2, 1, for geometric objects and cluster detection; 8, 7, 9] for sparse signals detection and identification; [11, 19] about density inference and [23, 20, [5, 6] for more recent results on block signal detection using the 
penalized scan and average likelihood ratio. However, most of the the previous research focus on the univariate case rather than multivariate case and the detection problem rather than the identification problem. 2 considers the detection of block signal in both the univariate and multivariate cases, but not the identification. Moreover, the results are actually not optimal unless the size of the block are on the smallest scale. For the block signal identification problem in the univariate case, in [16, the authors characterized the identifiable region under the assumption of Gaussian white noise and $\log \left|I_{n}^{*}\right|=o(\log n)$, where $n$ is the length of the sequence and $\left|I_{n}^{*}\right|$ is the length of the block signal. They also proposed the Likelihood Ratio Selector (LRS) procedure and established its optimality under the above assumptions. However, their result excludes the common and important situation where $\left|I_{n}^{*}\right|=n^{1-\beta}$ for $0<\beta<1$. In fact, it can be shown that LRS is not optimal in this situation. Moreover, LRS procedure needs to pre-specify a parameter $L$ which is some number greater than $\left|I_{n}^{*}\right|$. Such $L$ is not always easy to pre-specify and the misspecification may cause misidentification.

In this paper, we establish the block signal identification theory under a more general assumption which includes the case $\left|I_{n}^{*}\right|=n^{1-\beta}$ for $0<\beta<$ 1 in the univariate setting. The multivariate version of this problem is also considered. A computational efficient procedure based on the penalized scan statistic is proposed and its optimality is established under Gaussian white noise assumption in both the univariate and multivariate settings. We note that in our procedure, there is no unknown parameters that need to be prespecified. Moreover, our results show that the block signal detection and block signal identification have the same statistical difficulty in both the univariate and multivariate settings, although the latter seems to be more challenging than the former.

In addition, we consider in our paper several generalizations of the block signal identification problem. Firstly, we consider an extension to the case of multiple block signals. We show that under certain assumptions, our procedure remains optimal in identifying all block signals. Moreover, in the discussion section, we briefly consider the robust identification problem when the noise distribution is unspecified and discuss about the block signal identification under the exponential family setting.

The rest of the paper is organized as follows. In Section 2, our identification procedure is introduced in the univariate case and its optimality is established under Gaussian white noise. In Section 3 , we extend our theorem to the multidimensional case and we show that our procedure remains optimal in identifying rectangular signals in the hyper-rectangle. In Section 4, we consider the situation when there are multiple block signals. In Section [5, a simulation study is carried out to illustrate our previous results. In Section 6, we give a brief discussion about the identification under an unknown noise distribution and under the exponential family setting. We also discuss about some future research topics.

In the end of this section, we make some notations. For two series $a_{n}$ and $b_{n}$, we define $a_{n} \ll b_{n}$ if $a_{n}=o\left(b_{n}\right)$, or equivalently, $\frac{a_{n}}{b_{n}} \rightarrow 0$. We may use this notation and the small-o notation interchangeably. For a set of random 
variables $X_{n}$ and a corresponding set of constant $a_{n}$, we define $X_{n}=o_{p}\left(a_{n}\right)$ if $X_{n} / a_{n}$ converges to 0 in probability. Similarly, we define $X_{n}=O_{p}\left(a_{n}\right)$ if for any $\epsilon>0$, there exists a finite $M$ such that $P\left(\left|X_{n} / a_{n}\right|>M\right)<\epsilon$ for all $n$.

\section{Block signal identification under Gaussian white noise}

Let's first consider block signal identification in the univariate setting. Suppose we observe that

$$
Y_{i}=\mu \mathbf{1}_{I_{n}^{*}}(i)+Z_{i}, \quad i=1, \ldots, n
$$

where $Z_{i}$ are i.i.d standard normal random variables and unknown interval $I_{n}^{*}=$ $\left(j_{n}, k_{n}\right], \quad 0 \leq j_{n}<k_{n}<n$ and

$$
\mathbf{1}_{I_{n}^{*}}(i)=\left\{\begin{array}{ll}
1 & i \in I_{n}^{*} \\
0 & i \notin I_{n}^{*}
\end{array} .\right.
$$

$\mu$ is an unknown number and for simplicity we assume that $\mu$ is non-negative. If $\mu$ is non-positive, we can replace $Y_{i}$ by $-Y_{i}$. Our goal is to estimate the support $I_{n}^{*}$ in model (10) where $I_{n}^{*}=\emptyset$ means $\mu=0$.

For block signal detection, which is a testing problem, we want to maximize the power of the test while controlling the type I error. Similarly, in the corresponding block signal identification problem we want to approximately find the start and the end point of the block signal (when exists) with high probability while control the type I error. To give the definition of consistency, we introduce the following notation. Let $H_{0}$ denote the null case that there exists no signal in the sequence and $H_{1}$ denote the case where there exists a block signal $I_{n}^{*}$. Define the (Hamming) distance between two intervals $I_{1}$ and $I_{2}$ as $D\left(I_{1}, I_{2}\right)=1-\frac{\left|I_{1} \cap I_{2}\right|}{\sqrt{\left|I_{1}\right|\left|I_{2}\right|}}$, where we take the convention $\frac{0}{0}=0$. The definition of consistency for the block signal identification problem is given below.

Definition 1. We call a procedure $\mathcal{P}$ to be consistent if its estimated interval $\hat{I}_{n}$ satisfies

$$
P_{H_{0}}\left(\hat{I}_{n} \neq \emptyset\right) \leq \alpha
$$

and

$$
P_{H_{1}}\left(D\left(\hat{I}_{n}, I_{n}^{*}\right)>\delta_{n}\right) \rightarrow 0
$$

for some $\delta_{n}=o(1)$, where $\emptyset$ denotes the empty set and $\alpha$ denotes the significance level.

In this section, we focus on those $I_{n}^{*}$ satisfying the following property: there exists a $\kappa>0$, such that $\left|I_{n}^{*}\right| \ll n^{1-\kappa}$. This mild assumption includes all intervals with length $n^{1-\beta}$ for $0<\beta \leq 1$, but not those with length $n / \log n$. The set of intervals considered here greatly extends those in [16, in which the author requires $\log \left(\left|I_{n}^{*}\right|\right) \ll \log n$. 
Before giving the identification procedure, we first introduce the concept of the approximation set which is introduced in [19, 23, 6]. The idea of the approximation set is that we only need to consider intervals with endpoints on a grid as long as we can approximate each interval relatively well. In this section, we define our approximation set as below:

$$
\mathcal{I}_{\text {app }}=\bigcup_{\ell=1}^{\ell_{\max }} \mathcal{I}_{\text {app }}(\ell) \cup \mathcal{I}_{\text {small }}, \quad \text { where } \ell_{\text {max }}=\left\lfloor\log _{2} \frac{n}{\log n}\right\rfloor
$$

in which,

$$
\begin{gathered}
\mathcal{I}_{\text {app }}(\ell)=\left\{(j, k]: j, k \in\left\{i d_{\ell}, i=0,1, \ldots\right\} \text { and } m_{\ell}<k-j \leq 2 m_{\ell}\right\}, \\
\mathcal{I}_{\text {small }}=\left\{(j, k]: k-j \leq m_{\ell_{\text {max }}}\right\} .
\end{gathered}
$$

where $m_{\ell}=n 2^{-\ell}, d_{\ell}=\left\lceil\frac{m_{\ell}}{6 \sqrt{\ell}}\right\rceil$. A simple counting argument shows that $\left|\mathcal{I}_{\text {app }}(\ell)\right| \leq\left(\frac{n}{d_{\ell}}+1\right)\left(\frac{m_{\ell}}{d_{\ell}}+1\right) \leq 144 \ell 2^{\ell}$ for $\ell=1, \ldots, \ell_{\max }$ and $\left|\mathcal{I}_{\text {small }}\right| \leq 2 n \log n$. Thus $\left|\mathcal{I}_{\text {app }}\right| \leq \sum_{\ell=1}^{\ell_{\max }} 144 \ell 2^{\ell}+2 n \log n=O(n \log n)$.

Remark 1. 1) In fact we can let $d_{l}=\left\lceil\frac{m_{\ell}}{\ell^{\zeta}}\right\rceil$ for some $c>0$ and $\zeta \geq 0.5$. $c$ and $\zeta$ control the precision of the approximation set and the choice is a trade off between computational efficiency and approximation error: the larger the $c$ and $\zeta$, the better the approximation while the heavier the computation.

2) Some different approximation sets are also introduced in [18] and [2]. It is not clear whether those approximation sets can lead to the same optimal result.

Define $\boldsymbol{Y}(I)=\frac{\sum_{i \in I} Y_{i}}{\sqrt{|I|}}$. Our identification procedure, denoted by $\mathcal{P}_{n}$, works as follows: If $\max _{I \in \mathcal{I}_{a p p}}\left(\boldsymbol{Y}(I)-\sqrt{2 \log \frac{e n}{|I|}}\right)<\gamma_{n}(\alpha)$, where $\gamma_{n}(\alpha)$ is the $(1-\alpha)$ quantile of the null distribution of $\max _{I \in \mathcal{I}_{a p p}}\left(\mathbf{Y}(I)-\sqrt{2 \log \frac{e n}{|I|}}\right)$, we claim there exists no signal, i.e. $\hat{I}_{n}=\emptyset$. Otherwise, our estimated interval is

$$
\hat{I}_{n}=\underset{I \in \mathcal{I}_{\text {app }}}{\operatorname{argmax}}\left(\boldsymbol{Y}(I)-\sqrt{2 \log \frac{e n}{|I|}}\right) .
$$

By Boole's inequality, we can show that under the null distribution, $P_{n}:=$ $\max _{I \in \mathcal{I}_{\text {app }}}\left(\boldsymbol{Y}(I)-\sqrt{2 \log \frac{e n}{|I|}}\right)=O_{p}(1), \operatorname{so~} \lim \sup _{n \rightarrow \infty} \gamma_{n}(\alpha)<\infty$, see [19].

It can be shown that $\mathcal{P}_{n}$ is optimal for block signal identification. In fact, the procedure $\mathcal{P}_{n}$ is consistent in identifying the support $I_{n}^{*}$ whenever the signal is in the detectable region. We summarize this fact in the following theorem.

Theorem 2. Assume Model (11) and there exists a $\kappa>0$ such that $\left|I_{n}^{*}\right| \ll$ $n^{1-\kappa}$. If $\mu \geq\left(\sqrt{2 \log \frac{e n}{\left|I_{n}^{*}\right|}}+b_{n}\right) / \sqrt{\left|I_{n}^{*}\right|}$ with $b_{n} \rightarrow+\infty$, then our identification procedure $\mathcal{P}_{n}$ is consistent with any $1 \gg \delta_{n} \gg \sqrt{\log \log n} / \sqrt{\log n}$. In addition, this procedure can be computed in $O(n \log n)$ time. 
From Section 2 of [6],$\mu \geq\left(\sqrt{2 \log \frac{e n}{\left|I_{n}^{*}\right|}}+b_{n}\right) / \sqrt{\left|I_{n}^{*}\right|}$ with $b_{n} \rightarrow+\infty$ is necessary for any test to be consistent in detecting the signal $I_{n}^{*}$. Since the identification problem is more challenging than the corresponding detection problem, we can conclude that $\mu \geq\left(\sqrt{2 \log \frac{e n}{\left|I_{n}^{*}\right|}}+b_{n}\right) / \sqrt{\left|I_{n}^{*}\right|}$ with $b_{n} \rightarrow+\infty$ is necessary for any procedure to be consistent in identifying the signal $I_{n}^{*}$. Thus our procedure $\mathcal{P}_{n}$ is in fact optimal in block signal identification under our current setting.

We can view $\delta_{n}$ as the precision for the signal recovery. From Theorem 2 we know that for any $\delta_{n}=\phi_{n} \sqrt{\log \log n} / \sqrt{\log n}$ with $\phi_{n} \rightarrow \infty$ at any rate, we have

$$
P_{H_{1}}\left(D\left(\hat{I}_{n}, I_{n}^{*}\right)<\delta_{n}\right) \rightarrow 1 .
$$

Thus $\delta_{n}=\phi_{n} \sqrt{\log \log n} / \sqrt{\log n}$ is the upper bound for the (hamming) distance between our recovered signal and the true signal. As $\delta_{n} \rightarrow 0$, we can be sure that our recovered signal is sufficiently close to the true signal asymptotically.

We also note that the block signal identification problem has the same statistical difficulty as the corresponding detection problem, although the former one seems to be more challenging than latter one. In the computational aspect, although there are $O\left(n^{2}\right)$ number of possible intervals in the sequence, our procedure runs in $O(n \log n)$ time, almost linear in the number of observations.

Remark 2. 1) If we let $d_{l}=\left\lceil\frac{m_{\ell}}{c \ell \zeta}\right\rceil$ for some $c>0$ and $\zeta \geq 0.5$ in the definition of approximation set, then Theorem 2 still holds and the computational complexity is $O\left(n c^{2} \log ^{2 \zeta} n\right)$.

2) If instead we define $\gamma_{n}(\alpha)$ as the $(1-\alpha)$ quantile of the null distribution of $\max _{0 \leq j<k \leq n}\left(\boldsymbol{Y}((j, k])-\sqrt{2 \log \frac{e n}{k-j}}\right)$, Theorem 2 would still hold with the same bound on $\delta_{n}$. However, one would expect a faster simulation for critical values using the previous definition of $\gamma_{n}(\alpha)$.

We make the following comparison between our procedure and the LRS procedure in [16. (1) The LRS procedure requires the length of the signal $\left|I_{n}^{*}\right|$ to satisfy $\log \left|I_{n}^{*}\right|=o(\log n)$. In contrast, we allow $\left|I_{n}^{*}\right|=n^{1-\beta}$ for $0<\beta \leq 1$. (2) The identification boundary for LRS procedure is $\frac{\sqrt{2 \log n}}{\sqrt{I_{n}^{*}}}$, which is optimal only for signals with the smallest spatial extent. In contrast, the identification boundary for $\mathcal{P}_{n}$ is $\sqrt{2 \log \frac{e n}{\left|I_{n}^{*}\right|}} / \sqrt{\left|I_{n}^{*}\right|}$, which is optimal for a broad range of signals. (3) In the LRS procedure, one needs to pre-specify the parameter $L$ which is some number greater than the length of the signal. However, since the length of the signal is unknown, it is not always easy to determine $L$ and the misspecification of $L$ may cause misidentification. Although it is argued in [16] that $L$ could be sometimes easily selected, we would prefer a procedure without any unknown parameters. Our procedure $\mathcal{P}_{n}$ has no need to specify the parameter $L$ or any other unknown parameters. (4) The LRS procedure has a computational complexity of $O(n L)$. Depending on the choice of $L$, the complexity could be large. In contrast, our procedure $\mathcal{P}_{n}$ has a computational complexity of $O(n \log n)$, regardless of the length of the signal. We also note that besides LRS procedure in [16, similar multiscale methods also appear in 
2], 1] and [18. However, these methods do not lead to the same optimal results in our setting.

\section{Multi-dimensional rectangular signal identifi- cation}

In this case, we consider the problem of identifying the rectangular signal in the multi-dimensional hyper-rectangle.

Consider the $D$-dimensional model

$$
Y_{i}=\mu \mathbf{1}_{I_{n}^{*}}(i)+Z_{i}, \quad i=\left(i^{1}, \ldots, i^{D}\right) \in[1, \ldots, n]^{D}
$$

where $Z_{i}$ are i.i.d standard normal random variable and unknown rectangle

$$
I_{n}^{*}=\prod_{d=1}^{D}\left(j_{n, d}, k_{n, d}\right], \quad 0 \leq j_{n, d}<k_{n, d}<n, \quad \text { for each } d=1, \ldots D
$$

with sides parallel to the axes and with arbitrary sizes and aspect ratios. We denote the area of the hyper-rectangle by $\left|I_{n}^{*}\right|=\prod_{d=1}^{D}\left(k_{n, d}-j_{n, d}\right)$.

All of the results in this section can be easily extended to higher dimensions, but we will focus on the two-dimensional case $D=2$ to simplify our notation. We use the superscript $\{2\}$ to denote we are now considering the two-dimensional case.

We first introduce the approximation set for two-dimensional rectangle, which is a variation of the construction in [23. Using $\left(j_{1}, j_{2}, k_{1}, k_{2}\right)$ denotes the rectangle with vertices $\left(j_{1}, k_{1}\right),\left(j_{2}, k_{1}\right),\left(j_{2}, k_{2}\right)$ and $\left(j_{1}, k_{2}\right)$. For fixed $\ell$ and $0 \leq i \leq \ell$, define

$$
\begin{aligned}
\mathcal{I}_{a p p}^{\{2\}}(\ell, i)= & \left\{\left(j_{1}, j_{2}, k_{1}, k_{2}\right): j_{1}, j_{2} \in\left\{r^{(1)} d_{\ell, i}^{(1)}, r^{(1)}=0,1, \ldots\right\}\right. \\
& \text { and } k_{1}, k_{2} \in\left\{r^{(2)} d_{\ell, i}^{(2)}, r^{(2)}=0,1, \ldots\right\}, \\
& 0 \leq j_{1} \leq\left\lfloor\ell^{\frac{1}{2}} 2^{\ell-i}\right\rfloor, 1 \leq j_{2}-j_{1} \leq\left\lfloor\ell^{\frac{1}{2}}\right\rfloor, \\
& \left.0 \leq k_{1} \leq\left\lfloor\ell^{\frac{1}{2}} 2^{i}\right\rfloor, 1 \leq k_{2}-k_{1} \leq\left\lfloor 2 \ell^{\frac{1}{2}}\right\rfloor\right\}
\end{aligned}
$$

where $d_{\ell, i}^{(1)}=\left\lceil\ell^{-\frac{1}{2}} 2^{-\ell} 2^{i} n\right\rceil, d_{\ell, i}^{(2)}=\left\lceil\ell^{-\frac{1}{2}} 2^{-i} n\right\rceil$. Then let $\mathcal{I}_{a p p}^{\{2\}}(\ell)=\cup_{i=0}^{\ell} \mathcal{I}_{a p p}^{\{2\}}(\ell, i)$. Define $\ell_{\max }=\left\lfloor\log _{2} \frac{n^{2}}{\log n}\right\rfloor, m_{l}=n 2^{-\ell}$ and

$$
\mathcal{I}_{\text {small }}^{\{2\}}=\left\{I,|I| \leq m_{\ell_{\max }}\right\} .
$$

Then our approximation set $\mathcal{I}_{\text {app }}^{\{2\}}$ is defined as:

$$
\mathcal{I}_{\text {app }}^{\{2\}}=\bigcup_{\ell=1}^{\ell_{\max }} \mathcal{I}_{\text {app }}^{\{2\}}(\ell) \cup \mathcal{I}_{\text {small }}^{\{2\}}
$$

Define $\boldsymbol{Y}(I)=\frac{\sum_{i \in I} Y_{i}}{\sqrt{|I|}}$, now we are ready to introduce the property of the penalized scan statistic in the two-dimensional case in the following proposition. 
Proposition 3. Define

$$
P_{n}^{\{2\}}=\max _{I \in \mathcal{I}_{a p p}^{\{2\}}}\left(\boldsymbol{Y}(I)-\sqrt{2 \log \frac{e n^{2}}{|I|}}\right),
$$

Under the null hypothesis, $P_{n}^{\{2\}}=O_{p}(1)$, i.e. $P_{n}^{\{2\}}$ are uniformly bounded in probability.

We define $\gamma_{n}^{\{2\}}(\alpha)<\infty$ as the $(1-\alpha)$ quantile of the null distribution of $P_{n}^{\{2\}}$, which is well defined by Proposition 3 .

The identification procedure $\mathcal{P}_{n}^{\{2\}}$ in the two-dimensional case works as follows: If $\max _{I \in \mathcal{I}_{a p p}^{\{2\}}}\left(\mathbf{Y}(I)-\sqrt{2 \log \frac{e n^{2}}{|I|}}\right)<\gamma_{n}^{\{2\}}(\alpha)$, we claim there is no signal, i.e. $\hat{I}_{n}=\emptyset$. Otherwise, our estimated rectangle

$$
\hat{I}_{n}=\underset{I \in \mathcal{I}_{a p p}^{\{2\}}}{\operatorname{argmax}}\left(\boldsymbol{Y}(I)-\sqrt{2 \log \frac{e n^{2}}{|I|}}\right) .
$$

As in the one-dimensional case, one can establish the optimality for the above procedure in the two-dimensional case, which is given in the following theorem.

Theorem 4. Assume Model (5) and there exists a $\kappa>0$ such that $\left|I_{n}^{*}\right| \ll n^{2-\kappa}$. If $\mu \geq\left(\sqrt{2 \log \frac{e n^{2}}{\left|I_{n}^{*}\right|}}+b_{n}\right) / \sqrt{\left|I_{n}^{*}\right|}$ with $b_{n} \rightarrow+\infty$, then our procedure $\mathcal{P}_{n}^{\{2\}}$ is consistent with $1 \gg \delta_{n} \gg \sqrt{\log \log n} / \sqrt{\log n}$. In addition, this procedure can be computed in $O\left(n^{2} \log ^{2} n\right)$ time.

For a very similar argument as in Section 2 of [ [6] we can get $\mu \geq\left(\sqrt{2 \log \frac{e n^{2}}{\left|I_{n}^{*}\right|}}+\right.$ $\left.b_{n}\right) / \sqrt{\left|I_{n}^{*}\right|}$ is necessary for any test to be consistent in detecting the rectangular signal in the two-dimensional case and as a result $\mu \geq\left(\sqrt{2 \log \frac{e n^{2}}{\left|I_{n}^{*}\right|}}+b_{n}\right) / \sqrt{\left|I_{n}^{*}\right|}$ is also necessary for any procedure to be consistent in identifying the signal in the two-dimensional case. Thus our procedure is optimal under our current setting. Again, we conclude that in the two-dimensional case, the identification problem and the corresponding detection problem has the same statistical difficulty. Although there are $O\left(n^{4}\right)$ number of possible rectangles, our algorithm runs in $O\left(n^{2} \log ^{2} n\right)$, almost linear in the number of observations $n^{2}$. In general, in $d$ dimensional case, our algorithm runs in $O\left(n^{d} \log ^{d} n\right)$, almost linear in the number of observations $n^{d}$.

\section{Signal identification for multiple signals}

In the previous two sections, we focus on the situation where there exists only one signal. In this section, we consider the situation where there are multiple signals. We will only discuss the univariate case in this section for notation simplicity, but all our theory can be extended to multivariate case by using the 
corresponding approximation set. To further simplify the notation and avoid confusion, we suppress the subscript $n$ in $I_{n}^{*}$ in this section. We denote the set of true block signals $I^{*}=\left\{I_{1}^{*}, \ldots, I_{K}^{*}\right\}$, where $K$ is the number of block signals. Our model is:

$$
Y_{i}=\mu_{I^{*}}(i)+Z_{i}, \quad i=1, \ldots, n
$$

where $Z_{i}$ are i.i.d standard normal random variable, the unknown set of intervals $I^{*}=\left\{I_{1}^{*}, \ldots, I_{K}^{*}\right\}=\left\{\left(j_{1}, k_{1}\right], \ldots,\left(j_{K}, k_{K}\right], 0 \leq j_{1}<k_{1}<j_{2}<\ldots<k_{K} \leq n\right\}$ and

$$
\mu_{I^{*}}(i)=\left\{\begin{array}{ll}
\mu_{I_{j}^{*}} & i \in I_{j}^{*} \\
0 & \text { otherwise }
\end{array} .\right.
$$

In another word, $\mu_{I^{*}}(i)$ has constant value $\mu_{I_{j}^{*}}$ on each interval $I_{j}^{*}, j=1, \ldots K$ and 0 otherwise.

We give the following definition of consistency for the identification procedure for multiple block signals.

Definition 5. We call a procedure $\mathcal{P}$ is consistent in identifying multiple block signals if its estimated set of signals (intervals) $\hat{I}=\left\{\hat{I}_{1}, \ldots, \hat{I}_{\hat{K}}\right\}$ satisfies

$$
\begin{gathered}
P_{H_{0}}(\hat{I} \neq \emptyset) \leq \alpha \\
E_{H_{1}}(\hat{K}) \leq K+C(\alpha)+o(1)
\end{gathered}
$$

and

$$
P_{H_{1}}\left(\max _{j=1}^{K} \min _{i=1}^{\hat{K}} D\left(\hat{I}_{i}, I_{j}^{*}\right)>\delta_{n}\right) \rightarrow 0
$$

for some $\delta_{n}=o(1)$, where $\emptyset$ denotes the empty set, $\alpha$ denotes the significant level, $C(\alpha)>0$ is a function that depends on $\alpha$ and $\lim _{\alpha \rightarrow 0} C(\alpha)=0$.

Equation (9) in fact implies that $P_{H_{1}}(\hat{K} \geq K) \rightarrow 1$ as $n \rightarrow \infty$. Together with Equation (8), we can conclude that if a procedure is consistent asymptotically, it can identify all true intervals and may include some false intervals. In expectation, the number of the false intervals our procedure identifies goes to 0 as $\alpha \rightarrow 0$ and $n \rightarrow \infty$.

Note this slightly complicated definition reflects a fundamental difficulty in this problem: even after we correctly identifying all signals, we get back to the null cases and we cannot avoid a probability less than $\alpha$ that we include a false interval. One can take $\alpha$ to be small and Equation (7), (8) and (9) still hold so this effect is minimal.

Theorem 2 can be generalized to the current situation as long as $K=$ $O\left(\log ^{p} n\right)$ for some $p>0$. Here we also assume the minimum distance between two signals $d_{\text {min }} \gg \max _{j=1}^{K}\left|I_{j}^{*}\right| \log n$.

Define $\mathcal{I}_{\text {app }}$ and $\gamma_{n}(\alpha)$ as in Section 2, Our identification procedure, denote by $\mathcal{P}_{n, m u l t i}$ for multiple signals works as follows:

Initialize our result set $\hat{I}$ as empty set $\emptyset$. Denote $\mathcal{I}_{a p p}^{1}=\mathcal{I}_{a p p}$. 
Let $i=1$, repeat the following step until $\max _{I \in \mathcal{I}_{a p p}^{i}}\left(\mathbf{Y}(I)-\sqrt{2 \log \frac{e n}{|I|}}\right)<$ $\gamma_{n}(\alpha)$ :

\{

Denote $\hat{I}_{i}=\operatorname{argmax}_{I \in \mathcal{I}_{a p p}^{i}}\left(\mathbf{Y}(I)-\sqrt{2 \log \frac{e n}{|I|}}\right)$, then set $\hat{I}=\hat{I} \cup \hat{I}_{i}$,

Let $\mathcal{I}_{a p p}^{i+1}=\mathcal{I}_{a p p}^{i} \backslash\left\{I \in \mathcal{I}_{a p p}^{i}: I \cap \hat{I}_{i} \neq \emptyset\right\}$.

Increase $i$ by 1 .

\}

We have the following theorem regarding the consistency of the procedure $\mathcal{P}_{n, \text { multi }}$.

Theorem 6. Assume Model (6) and there exists a $\kappa>0$ such that $\left|I_{j}^{*}\right| \ll n^{1-k}$ for each $j=1, \ldots, K$ and the minimum distance between two signals $d_{\text {min }} \gg$ $\max _{j=1}^{K}\left|I_{j}^{*}\right| \log n$ and $K=O\left(\log ^{p} n\right)$ for some $p>0$, if for each $j=1, \ldots, K$, $\mu_{I_{j}^{*}} \geq\left(\sqrt{2 \log \frac{e n}{\left|I_{j}^{*}\right|}}+b_{n, j}\right) / \sqrt{\left|I_{j}^{*}\right|}$ with $b_{n, j} / \sqrt{\log \log n} \rightarrow+\infty$, then our procedure $\mathcal{P}_{n, \text { multi }}$ is consistent with $1 \gg \delta_{n} \gg \sqrt{\log \log n} / \sqrt{\log n}$.

The main difference here compare to Theorem 2 is we require $b_{n, j} / \sqrt{\log \log n} \rightarrow$ $\infty$ rather than $b_{n, j} \rightarrow \infty$. This stronger requirement will ensure that the probability of making mistakes for every iteration is small enough so that the identification procedure is still consistent after all iterations. The reason we assume that the minimum spacing between the intervals $d_{\min } \gg \max _{j=1}^{K}\left|I_{j}^{*}\right| \log n$ is that if two intervals are very close, it is difficult to identify the exact location of the signal under the influence of another signal. For example, with nondiminishing probability that two intervals both with length $\left|I^{*}\right|$ and distance $\left|I^{*}\right|$ will be identified as one signal with length about $3\left|I^{*}\right|$. This is different from the detection problem where no such requirement is needed.

As a special case, if all signals are of the same length $\left|I_{1}^{*}\right|$, an explicit lower bound can be given.

Theorem 7. Assume Model (6), and $K=O\left(\log ^{p} n\right)$, if all signals are of the same length $\left|I_{1}^{*}\right|$, then no procedure is consistent if $\mu_{I_{j}^{*}}<\sqrt{2 \log \frac{e n}{\left|I_{1}^{*}\right|}} / \sqrt{\left|I_{1}^{*}\right|}$. Thus our procedure $\mathcal{P}_{n, \text { multi }}$ is in fact optimal under the above assumptions.

When $\mu_{I_{j}^{*}}<\sqrt{2 \log \frac{e n}{\left|I_{1}^{*}\right|}} / \sqrt{\left|I_{1}^{*}\right|}$, then an algorithm would either fail to discover all signals and/or will include too may false discoveries, see Lemma 1 in [16] and more details in Theorem 5 in [14.

Remark 3. If the true number of signals $K$ is known, we can stop the procedure $\mathcal{P}_{n, \text { multi }}$ after $K$ iterations. It can be shown that this modified procedure is still consistent and its estimated set does not contain any false intervals.

\section{Simulation Study}

We have shown that by adopting the correct penalty term, the procedure based on the penalized scan can be much more powerful, which is the major difference 
Figure 1: Simulated similarities for different $\mu$. The left sub-graph gives the result for $\left|I_{n}^{*}\right|=1000$ and the right sub-graph gives the result for $\left|I_{n}^{*}\right|=100$. In both graphs, the black solid line denotes the penalized procedure $\mathcal{P}_{n}$ and the right dashed line denotes the unpenalized procedure $\mathcal{P}_{n}^{\text {unpen }}$. The $x$-axis is $\mu \sqrt{\left|I^{*}\right|}$ and $y$-axis is similarity.
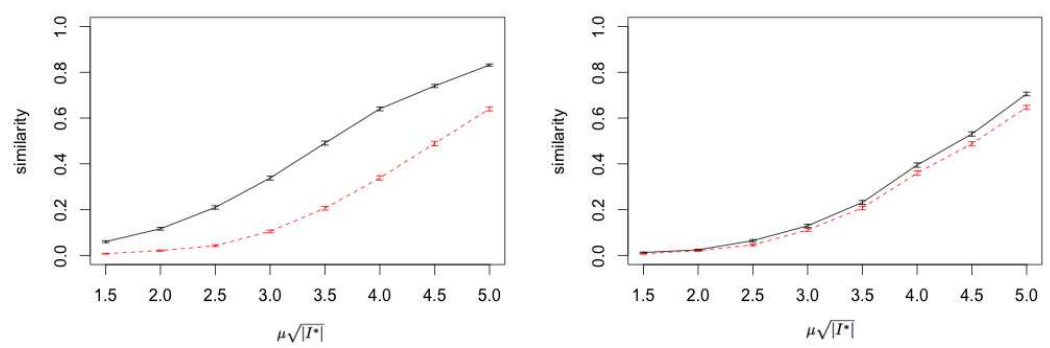

between our procedure and the LRS procedure in [16. It would be difficult to compare these two procedures directly since LRS needs to specify additional parameter $L$ and its optimality is established under some additional assumptions. It is also unclear how LRS works in high-dimension. So in this section, we will illustrate the previous results with a simulation study for Model (1) by comparing the performance of the identification procedure $\mathcal{P}_{n}$ defined in Section 2 and the identification procedure without the penalty term, denote by $\mathcal{P}_{n}^{\text {unpen }}$. To be comparable, $\mathcal{P}_{n}^{\text {unpen }}$ is defined exactly the same on the same approximation set except we set the penalty term to 0 instead of $\sqrt{2 \log \frac{e n}{|I|}}$. It can be shown that the identification boundary for $\mathcal{P}_{n}^{\text {unpen }}$ is $\sqrt{2 \log n} / \sqrt{\left|I_{n}^{*}\right|}$, which is the same as LRS. If $\left|I_{n}^{*}\right|=n^{1-p}, p \in(0,1)$, this detection boundary is $p^{-1 / 2}$ times larger than the optimal boundary.

Denote the (Hamming) similarity between interval $I_{1}$ and $I_{2}$ by $1-D\left(I_{1}, I_{2}\right)=$ $\frac{\left|I_{1} \cap I_{2}\right|}{\sqrt{\left|I_{1}\right|\left|I_{2}\right|}}$ and $\gamma_{n}(\alpha)$ as in Section2, For a particular simulation, if $\max _{I \in \mathcal{I}_{\text {app }}}(\boldsymbol{Y}(I)-$ $\left.\sqrt{2 \log \frac{e n}{|I|}}\right)>\gamma_{n}(\alpha)$, the similarity is calculated as $\frac{\left|\hat{I}_{n} \cap I_{n}^{*}\right|}{\sqrt{\left|\hat{I}_{n}\right|\left|I_{n}^{*}\right|}}$, where $\hat{I}_{n}$ is defined in (44); otherwise the similarity is set to be 0 . We do the same for $\mathcal{P}_{n}^{\text {unpen }}$, except that $\gamma_{n}(\alpha)$ is replaced by $\tau_{n}(\alpha)$, which is defined as the $(1-\alpha)$ quantile of $\max _{I \in \mathcal{I}_{a p p}} \boldsymbol{Y}(I)$. In all of our simulations for univariate setting, we choose $n=10000$.

For the first simulation, we give the similarity for different choices of $\mu$ range from 1.5 to 5 with a step of 0.5 and signal length $\left|I_{n}^{*}\right|=100$ and $\left|I_{n}^{*}\right|=1000$, respectively. The result is given in Figure 1. From Figure 1, we can see that when $\left|I_{n}^{*}\right|=1000$, which is relatively large, $\mathcal{P}_{n}$ performs much better than $\mathcal{P}_{n}^{\text {unpen }}$, while when $\left|I_{n}^{*}\right|=100$, which is relatively small, the performance of $\mathcal{P}_{n}$ is only slightly better.

For the second simulation, we give the similarities for different choices of the 
Figure 2: Simulated similarities for penalized procedure (black solid line) and unpenalized procedure (red dashed line) under different ratios $n /\left|I_{n}^{*}\right|$ and $\mu \sqrt{\left|I^{*}\right|}=1.2 * \sqrt{2 \log \frac{e n}{\left|I_{n}^{*}\right|}}+0.1$. The $x$-axis is the ratio in log-scale and $y$-axis is the similarity.

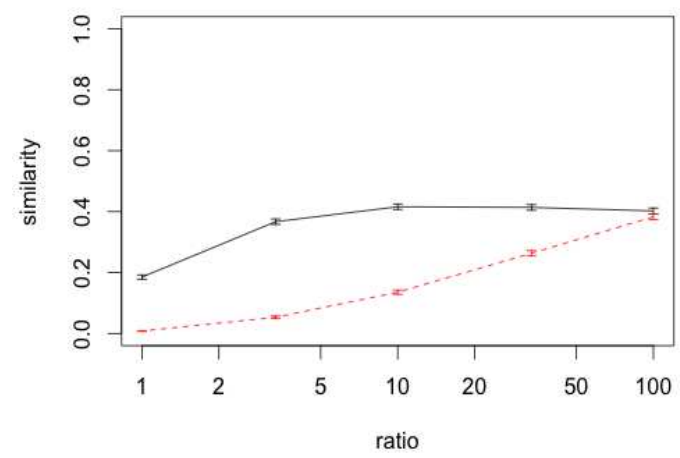

ratio $n /\left|I_{n}^{*}\right|$. For each choice of $n /\left|I_{n}^{*}\right|$, we choose $\mu \sqrt{\left|I^{*}\right|}=1.2 * \sqrt{2 \log \frac{e n}{\left|I_{n}^{*}\right|}}+0.1$. The simulation result is shown in Figure 2, We can see that the $\mathcal{P}_{n}^{\text {unpen }}$ seems to be powerless when $n /\left|I_{n}^{*}\right|$ is small $\left(\left|I_{n}^{*}\right|\right.$ is large). However, the gap between two procedures becomes smaller as the ratio $n /\left|I_{n}^{*}\right|$ increase.

We also compared the performance for Model (5) in the two-dimensional case between our procedure $\mathcal{P}_{n}^{\{2\}}$ defined in Section 3 and $\mathcal{P}_{n}^{\{2\}, \text { unpen, which }}$ is defined exactly the same on the same approximation set except the penalty term is set to 0 . The simulation result is shown in Figure 3, In this simulation, we give the similarity for different choices of $\mu$ range from 2.5 to 6 with a step of 0.5 . We choose $n=100$, so our space is a $100 \times 100$ rectangle. The left sub-graph gives the result for rectangular signal with width 30 and height 40 while the right sub-graph gives the result for rectangular signal with width 15 and height 80 . Note that the area of the rectangle $\left|I_{n}^{*}\right|=1200$ in both cases but the aspect ratios are different. We can see that $\mathcal{P}_{n}^{\{2\}}$ performs much better than $\mathcal{P}_{n}^{\{2\}, \text { unpen }}$ in both cases and the performance is robust with respect to different aspect ratios.

All similarities in Figure1, 2and 3 are with respect to a 5\% significance level. The critical values were simulated with 10000 Monte Carlo samples, and the similarities were simulated with 2000 Monte Carlo samples. The location of the signal was sampled at random in each of these simulations to avoid confounding with the approximation scheme. 
Figure 3: Simulated similarities for different $\mu$ in a $100 \times 100$ rectangle. The left sub-graph gives the result for $\left|I_{n}^{*}\right|=30 \times 40$ and the right sub-graph gives the result for $\left|I_{n}^{*}\right|=15 \times 60$. In both graphs, the black solid line denotes the penalized procedure $\mathcal{P}_{n}^{\{2\}}$ and the right dashed line denotes the unpenalized procedure $\mathcal{P}_{n}^{\{2\} \text {,unpen }}$. The $x$-axis is $\mu \sqrt{\left|I^{*}\right|}$ and $y$-axis is similarity.
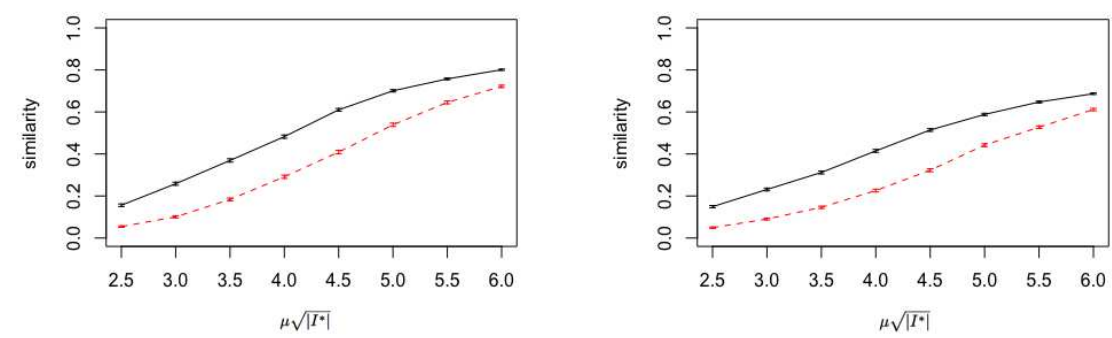

\section{Extensions and Discussion}

\subsection{Identification under the exponential family}

Now we consider the block signal identification problem under the exponential family setting. If instead of Gaussian noise model, we observe independent random variables $Y_{i}, i=1, \ldots, n$ through the one-dimensional exponential family model $Y_{i} \sim F_{\nu_{i}}, i=1, \ldots, n$, where

$$
\nu_{i}=a+b \mathbf{1}_{I_{n}^{*}}(i)
$$

with baseline $a$ known, signal strength $b$ unknown, and unknown interval $I_{n}^{*}$ defined the same as in Model (1). The task is to recover the support of $I_{n}^{*}$. If $F$ is the standard Gaussian distribution with $a=0$, we get back to our Gaussian noise Model (11). Notice that if $|I|$ is sufficiently large, then under $H_{0}, \boldsymbol{Y}(I)$ would be approximately normally distributed, which suggest the optimality results in Section 2 would still hold provided $\left|I_{n}^{*}\right|$ is large enough. Formally, if $\left|I_{n}^{*}\right| \geq \log ^{3+\delta} n$ for some $\delta>0$ and denote $\mathcal{I}_{a p p}^{\text {exp }}=\mathcal{I}_{\text {app }} \cap\left\{I:|I| \geq \log ^{3+\delta} n\right\}$, then our identification procedure in Section 2 is consistent by replacing $\mathcal{I}_{a p p}$ with $\mathcal{I}_{\text {app }}^{\exp }$. For some similar arguments, see [12] and [1]. Alternatively, one can use $\sqrt{2 \log \boldsymbol{T}(I)}$ instead of $\boldsymbol{Y}(I)$ where $\boldsymbol{T}(I)$ is the local likelihood ratio statistic for testing $H_{0}: b=0$ against $H_{1}: b \neq 0$ on interval $I$.

\subsection{Identification with unspecified noise distribution}

In [22], the author consider the identification problem with an unspecified noise distribution. The idea is to apply the identification procedure LRS on the "local median transformed" data. For more details about local median transformation, we refer the reader to [3]. It is worthwhile to note that our procedures $\mathcal{P}_{n}, \mathcal{P}_{n}^{\{2\}}$, 
$\mathcal{P}_{n, \text { multi }}$ are also adapted to the local median transformation and would give a near-optimal solution over a broad range of noise distribution with a much milder assumption on the length of the signal.

\subsection{Disucssion}

It is also interesting to compare our results with other results in change-point detection settings. For most research in change-point detection area, they typically seek to find an rate optimal solution rather than an exact optimal solution, due to the more complex structure they consider, see for example [12] and [4]. Our signal identification problem has a slightly easier setting and we can achieve the exact optimal constant. We have shown in the simulation that the constant actually matters and a suboptimal constant may lead to a significant loss of power. Last but not the least, in Theorem 7 , we assume that the number of block signals $K=O\left(\log ^{p} n\right)$ for some $p>0$. If instead we assume $K=n^{\delta}$ for some $\delta>0$, then our procedure may not be optimal anymore. In fact, in this case, block signal identification would be statistically more difficult than the block signal detection, as there are so many block signals. It would be interesting to develop an optimality theory under this situation, which is left as an open problem.

\section{Acknowledgements}

The author thanks Professor G. Walther for supervision and some helpful suggestions. We also owe thanks to the referees for a number of corrections and suggestions.

\section{Appendix}

\section{A.1 Proofs for Section 2}

Before giving the proof of Theorem 2 we first introduce some useful lemmas.

The following lemma is proved in [6], which is a consequence of a result in 10.

Lemma 8. Define $\boldsymbol{Z}_{n}(I)=\frac{\sum_{i \in I} Z_{i}}{\sqrt{|I|}}$. Let $J \in \mathcal{I}_{n}$, where $\mathcal{I}_{n}=\{(j, k], 0 \leq j<$ $k \leq n\}$ and $J$ does not depend on $\boldsymbol{Z}_{n}$. Then

$$
\max _{I \in \mathcal{I}_{n}: I \subset J}\left(Z_{n}(I)-\sqrt{2 \log \frac{e|J|}{|I|}}\right) \stackrel{d}{=}: L<\infty \quad \text { a.s. }
$$

here the random variable $L$ defined above is universally applicable for all $J$ and $n$ and is finite almost surely. 
Throughout the proof of this paper, we ignore the rounding issues in the definition of $d_{\ell}$ whenever this does not affect our results. To simplify the notation, in the following proof we use $I^{*}$ rather than $I_{n}^{*}$ to denote the true signal whenever this does not cause confusion.

The following lemma shows that we can approximate every interval well using our approximation set $\mathcal{I}_{\text {app }}$ defined in Section 2.

Lemma 9. For each $I^{*}$ with $\left|I^{*}\right| \ll n$, there exists an $\tilde{I} \in \mathcal{I}_{\text {app }}$ such that $D\left(\tilde{I}, I^{*}\right) \leq \frac{1}{3 \sqrt{\log _{2} \frac{n}{\left|I^{*}\right|}}}$ and $\sqrt{2 \log \frac{e n}{|\tilde{I}|}}-\sqrt{2 \log \frac{e n}{\left|I^{*}\right|}}=o(1)$.

Proof. There are two cases: $\left|I^{*}\right|>m_{\ell_{\max }}$ and $\left|I^{*}\right| \leq m_{\ell_{\max }}$.

Consider first when $\left|I^{*}\right|>m_{\ell_{\max }}$. Let $\ell^{*}$ be the integer satisfying $m_{\ell^{*}}<$ $\left|I^{*}\right| \leq 2 m_{\ell^{*}}$. By our construction, unless $\left|I^{*}\right|<m_{\ell^{*}}+2 d_{\ell^{*}}$, there exists an interval $\tilde{I} \in \mathcal{I}_{\text {app }}$, such that $\tilde{I} \subset I^{*}$ and

$$
|\tilde{I}|>\left(1-\frac{2 d_{\ell^{*}}}{m_{\ell^{*}}}\right)\left|I^{*}\right|=\left(1-\frac{1}{3 \sqrt{\ell^{*}}}\right)\left|I^{*}\right| \geq\left(1-\frac{1}{3 \sqrt{\log _{2} \frac{n}{\left|I^{*}\right|}}}\right)\left|I^{*}\right| .
$$

Thus

$$
D\left(\tilde{I}, I^{*}\right)=1-\sqrt{\frac{|\tilde{I}|}{\left|I^{*}\right|}} \leq 1-\sqrt{1-\frac{1}{3 \sqrt{\log _{2} \frac{n}{I^{*} \mid}}}} \leq \frac{1}{3 \sqrt{\log _{2} \frac{n}{I^{*} \mid}}}
$$

and

$$
\begin{aligned}
0 & \leq \sqrt{2 \log \frac{e n}{|\tilde{I}|}}-\sqrt{2 \log \frac{e n}{\left|I^{*}\right|}} \\
& \leq \sqrt{2 \log \frac{e n}{\left|I^{*}\right|\left(1-\frac{1}{3 \sqrt{\log _{2} \frac{n}{\left|I^{*}\right|}}}\right)}}-\sqrt{2 \log \frac{e n}{\left|I^{*}\right|}} \\
& \leq \frac{-\log \left(1-\frac{1}{3 \sqrt{\log _{2} \frac{n}{\left|I^{*}\right|}}}\right)}{\sqrt{2 \log \frac{e n}{\left|I^{*}\right|}}}
\end{aligned}
$$

As we assume $\left|I^{*}\right| \ll n$, then numerator goes to zero and the denominator goes to infinity, so $\sqrt{2 \log \frac{e n}{|\tilde{I}|}}-\sqrt{2 \log \frac{e n}{\left|I^{*}\right|}}=o(1)$.

If $\left|I^{*}\right|<m_{\ell^{*}}+2 d_{\ell^{*}}$, then there exists an interval $\tilde{I} \in \mathcal{I}_{a p p}$, such that $\tilde{I} \supset I^{*}$,

$$
|\tilde{I}|<\left(1+\frac{2 d_{\ell^{*}}}{m_{\ell}^{*}}\right)\left|I^{*}\right|=\left(1+\frac{1}{3 \sqrt{\ell^{*}}}\right)\left|I^{*}\right| \leq\left(1+\frac{1}{3 \sqrt{\log _{2} \frac{n}{\left|I^{*}\right|}}}\right)\left|I^{*}\right| .
$$

The remaining proof for bounding $D\left(\tilde{I}, I^{*}\right)$ and $\sqrt{2 \log \frac{e n}{|\tilde{I}|}}-\sqrt{2 \log \frac{e n}{\left|I^{*}\right|}}$ are similar to the case $\tilde{I} \subset I^{*}$.

Now consider when $\left|I^{*}\right| \leq m_{\ell_{\max }}$, then $I^{*} \in \mathcal{I}_{a p p}$, and thus we can simply let $\tilde{I}=I^{*}$ and the theorem holds trivially. 


\section{Proof of Theorem 2,}

Proof. Denote $\boldsymbol{X}(I)=\boldsymbol{Y}(I)-\sqrt{2 \log \frac{e n}{T T}}$ for interval $I$.

By the definition of $\gamma_{n}(\alpha)$,

$$
P_{H_{0}}(I \neq \emptyset)=P_{H_{0}}\left(\max _{I \in \mathcal{I}_{\text {app }}} \boldsymbol{X}(I)>\gamma_{n}(\alpha)\right) \leq \alpha,
$$

which proves equation (2).

Now turn to prove equation (3). Define

$$
K_{0}=\left\{I \in \mathcal{I}_{\text {app }}: I \cap I^{*}=\emptyset\right\}
$$

and

$$
K_{1}=\left\{I \in \mathcal{I}_{a p p}: I \cap I^{*} \neq \emptyset \text { and } D\left(I, I^{*}\right)>\delta_{n}\right\} .
$$

By Lemma 9, there exists an $\tilde{I} \in \mathcal{I}_{a p p}$ such that $D\left(\tilde{I}, I^{*}\right) \leq \frac{1}{\sqrt[3]{\log _{2} \frac{n}{I^{*}}}}$ and $\sqrt{2 \log \frac{e n}{|\tilde{I}|}}-\sqrt{2 \log \frac{e n}{\left|I^{*}\right|}}=o(1)$. Then,

$$
\begin{aligned}
P_{H_{1}}\left(D\left(\hat{I}, I^{*}\right)>\delta_{n}\right) & \leq P_{H_{1}}\left(\max \left(\max _{I \in \mathcal{I}_{a p p}, D\left(I, I^{*}\right)>\delta_{n}} \boldsymbol{X}(I), \gamma_{n}(\alpha)\right) \geq \boldsymbol{X}(\tilde{I})\right) \\
& \leq P_{H_{1}}\left(\max _{I \in K_{0}} \boldsymbol{X}(I) \geq \boldsymbol{X}(\tilde{I})\right)+P_{H_{1}}\left(\max _{I \in K_{1}} \boldsymbol{X}(I) \geq \boldsymbol{X}(\tilde{I})\right)+P_{H_{1}}\left(\gamma_{n}(\alpha) \geq \boldsymbol{X}(\tilde{I})\right) .
\end{aligned}
$$

By Lemma $8 \max _{I \in K_{0}} \boldsymbol{X}(I) \stackrel{d}{\leq} L<\infty$ a.s. and $\lim _{\sup } \sup _{n \rightarrow \infty} \gamma_{n}(\alpha)<\infty$ a.s..

Also notice that under $H_{1}$, by Lemma 9 , we have

$$
\begin{aligned}
\boldsymbol{X}(\tilde{I}) & =\boldsymbol{Y}(\tilde{I})-\sqrt{2 \log \frac{e n}{|\tilde{I}|}} \\
\geq & -|\boldsymbol{Z}(\tilde{I})|+\left(1-D\left(\tilde{I}, I^{*}\right)\right)\left(\sqrt{2 \log \frac{e n}{\left|I^{*}\right|}}+b_{n}\right)-\sqrt{2 \log \frac{e n}{|\tilde{I}|}} \\
\geq & -|\boldsymbol{Z}(\tilde{I})|+\left(1-D\left(\tilde{I}, I^{*}\right)\right) b_{n}-\left(\sqrt{2 \log \frac{e n}{|\tilde{I}|}}-\sqrt{2 \log \frac{e n}{\left|I^{*}\right|}}\right)-D\left(\tilde{I}, I^{*}\right) \sqrt{2 \log \frac{e n}{\left|I^{*}\right|}} \\
& \geq-|\boldsymbol{Z}(\tilde{I})|+\left(1-D\left(\tilde{I}, I^{*}\right)\right) b_{n}-\frac{\sqrt{2 \log \frac{e n}{\left|I^{*}\right|}}}{3 \sqrt{\log _{2} \frac{n}{\left|I^{*}\right|}}}-o(1) \\
& \stackrel{p}{\rightarrow} \infty
\end{aligned}
$$

so

$$
P_{H_{1}}\left(\max _{I \in K_{0}} \boldsymbol{X}(I) \geq \boldsymbol{X}(\tilde{I})\right) \rightarrow 0
$$

and

$$
P_{H_{1}}\left(\gamma_{n}(\alpha) \geq \boldsymbol{X}(\tilde{I})\right) \rightarrow 0 .
$$

Denote

$$
K_{\text {near }}=\left\{I \in K_{1}: \frac{|I|}{\log n} \leq\left|I^{*}\right| \leq|I| \log n\right\} .
$$


To finish the proof, it remains to show that $P_{H_{1}}\left(\max _{I \in K_{1}} \boldsymbol{X}(I) \geq \boldsymbol{X}(\tilde{I})\right) \rightarrow 0$.

First notice that

$$
\begin{aligned}
P_{H_{1}}\left(\max _{I \in K_{1}} X(I) \geq X(\tilde{I})\right) & \leq \sum_{I \in K_{\text {near }}} P_{H_{1}}(\boldsymbol{X}(I) \geq \boldsymbol{X}(\tilde{I}))+P_{H_{1}}\left(\max _{I \in K_{1} \backslash K_{\text {near }}} \boldsymbol{X}(I) \geq \boldsymbol{X}(\tilde{I})\right) \\
=: & (A)+(B)
\end{aligned}
$$

We need the following two lemmas to bound part (A) and (B), respectively. The proof of these lemmas is given after this theorem.

Lemma 10.

\section{Lemma 11.}

$$
\sum_{I \in K_{\text {near }}} P_{H_{1}}(\boldsymbol{X}(I) \geq \boldsymbol{X}(\tilde{I})) \rightarrow 0
$$

$$
P_{H_{1}}\left(\max _{I \in K_{1} \backslash K_{\text {near }}} \boldsymbol{X}(I) \geq \boldsymbol{X}(\tilde{I})\right) \rightarrow 0 .
$$

Combining Lemma 10 and 11, we see that $P_{H_{1}}\left(\max _{I \in K_{1}} X(I) \geq X(\tilde{I})\right) \rightarrow 0$. This together with Equations (10) and (11) will lead to $P_{H_{1}}\left(D\left(\hat{I}, I^{*}\right)>\delta_{n}\right) \rightarrow 0$ and we finish our proof.

\section{Proof of Lemma 10:}

Proof. For each interval $I \in K_{\text {near }}$, we have

$$
\begin{aligned}
& P_{H_{1}}(\boldsymbol{X}(I) \geq \boldsymbol{X}(\tilde{I})) \\
= & P_{H_{1}}\left(\boldsymbol{Y}(I)-\boldsymbol{Y}(\tilde{I}) \geq \sqrt{2 \log \frac{e n}{|I|}}-\sqrt{2 \log \frac{e n}{|\tilde{I}|}}\right) .
\end{aligned}
$$

Simple calculation shows that $\boldsymbol{Y}(I)-\boldsymbol{Y}(\tilde{I})$ has a normal distribution with mean

$$
-\mu \sqrt{\left|I^{*}\right|}\left(D\left(I, I^{*}\right)-D\left(\tilde{I}, I^{*}\right)\right)
$$

and variance

$$
2 D(I, \tilde{I}) \leq 2
$$

Thus,

$$
\begin{aligned}
& \sum_{I \in K_{\text {near }}} P_{H_{1}}(\boldsymbol{X}(I) \geq \boldsymbol{X}(\tilde{I})) \\
\leq & \sum_{I \in K_{\text {near }}} \bar{\Phi}\left(\left(\left(\mu \sqrt{\left|I^{*}\right|}\left(D\left(I, I^{*}\right)-D\left(\tilde{I}, I^{*}\right)\right)\right)-\sqrt{2 \log \frac{e n}{|\tilde{I}|}}+\sqrt{2 \log \frac{e n}{|I|}}\right) / \sqrt{2}\right)
\end{aligned}
$$

where $\bar{\Phi}$ denotes the upper cumulative distribution function of the normal distribution.

Under the assumption that $\delta_{n} \gg \sqrt{\log \log n} / \sqrt{\log n}$ and $b_{n} \rightarrow+\infty$, we have

$$
\left(\delta_{n}-\frac{1}{3 \sqrt{\log _{2} \frac{n}{\left|I^{*}\right|}}}\right)\left(\sqrt{2 \log \frac{e n}{\left|I^{*}\right|}}+b_{n}\right) \gg \sqrt{\log \log n} .
$$


Note that $D\left(I, I^{*}\right)>\delta_{n}$ in $K_{1}$ and $D\left(\tilde{I}, I^{*}\right) \leq \frac{1}{\sqrt[3]{\log _{2} \frac{n}{\left|I^{*}\right|}}}$ and $\mu \sqrt{\left|I^{*}\right|} \geq$ $\sqrt{2 \log \frac{e n}{I^{*} \mid}}+b_{n}$ so

$$
\mu \sqrt{\left|I^{*}\right|}\left(D\left(I, I^{*}\right)-D\left(\tilde{I}, I^{*}\right)\right) \gg \sqrt{\log \log n}
$$

When $I \in K_{\text {near }}$, we have $\frac{|I|}{\log n} \leq\left|I^{*}\right| \leq|I| \log n$, then

$$
\begin{aligned}
\left|\sqrt{2 \log \frac{e n}{|I|}}-\sqrt{2 \log \frac{e n}{|\tilde{I}|}}\right| & \leq\left|\sqrt{2 \log \frac{e n}{\left|I^{*}\right|}}-\sqrt{2 \log \frac{e n}{|\tilde{I}|}}\right|+\left|\sqrt{2 \log \frac{e n}{\left|I^{*}\right|}}-\sqrt{2 \log \frac{e n}{|I|}}\right| \\
& \leq\left|\sqrt{2 \log \frac{e n}{\left|I^{*}\right|}}-\sqrt{2 \log \frac{e n}{|\tilde{I}|}}\right|+\frac{2 \log \log n}{\sqrt{2 \log \frac{e n}{\left|I^{*}\right|}}}
\end{aligned}
$$

Since we assume there exists a $\kappa>0$, such that $\left|I^{*}\right| \ll n^{1-\kappa}$, then $\frac{2 \log \log n}{\sqrt{2 \log \frac{e n}{I^{*} \mid}}} \rightarrow$ 0. By Lemma 9, $\mid \sqrt{2 \log \frac{e n}{\left|I^{*}\right|}}-\sqrt{2 \log \frac{e n}{|\tilde{I}|} \mid}=o(1)$.

$$
\left|\sqrt{2 \log \frac{e n}{|I|}}-\sqrt{2 \log \frac{e n}{|\tilde{I}|}}\right|=o(1)
$$

Combine (12), (13) and using the inequality $\bar{\Phi}(x) \leq \exp \left(-\frac{x^{2}}{2}\right)$ for $x>1$, we have,

$\bar{\Phi}\left(\left(\left(\mu \sqrt{\left|I^{*}\right|}\left(D\left(I, I^{*}\right)-D\left(\tilde{I}, I^{*}\right)\right)\right)-\sqrt{2 \log \frac{e n}{|\tilde{I}|}}+\sqrt{2 \log \frac{e n}{|I|}}\right) / \sqrt{2}\right) \leq \log ^{-\eta} n$

for all $\eta>0$.

Simple counting shows the cardinality of the set $K_{\text {near }}$ is $O\left(\log ^{2} n\right)$. Thus

$$
\sum_{I \in K_{\text {near }}} P_{H_{1}}\left(\boldsymbol{X}(I) \geq \boldsymbol{X}\left(I^{*}\right)\right) \rightarrow 0
$$

\section{Proof of Lemma 11;}

Proof. When $I \in K_{1} \backslash K_{\text {near }}$, we have $1-D\left(I, I^{*}\right) \leq \min \left(\sqrt{\frac{|I|}{\left|I^{*}\right|}}, \sqrt{\frac{\left|I^{*}\right|}{|I|}}\right) \leq$ $1 / \sqrt{\log n}$. 
Thus,

$$
\begin{aligned}
& P_{H_{1}}\left(\max _{I \in K_{1} \backslash K_{\text {near }}} \boldsymbol{X}(I) \geq \boldsymbol{X}(\tilde{I})\right) \\
& =P_{H_{1}}\left(\max _{I \in K_{1} \backslash K_{\text {near }}}\left(\boldsymbol{Z}(I)+\left(1-D\left(I, I^{*}\right)\right)\left(\sqrt{2 \log \frac{e n}{\left|I^{*}\right|}}+b_{n}\right)-\sqrt{2 \log \frac{e n}{|I|}}\right)\right. \\
& \left.\left.\geq \boldsymbol{Z}(\tilde{I})+\left(1-D\left(\tilde{I}, I^{*}\right)\right)\left(\sqrt{2 \log \frac{e n}{\left|I^{*}\right|}}+b_{n}\right)-\sqrt{2 \log \frac{e n}{|\tilde{I}|}}\right)\right) \\
& \leq P_{H_{1}}\left(\max _{I \in K_{1} \backslash K_{\text {near }}}\left(\boldsymbol{Z}(I)-\sqrt{2 \log \frac{e n}{|I|}}+\frac{1}{\sqrt{\log n}}\left(\sqrt{2 \log \frac{e n}{\left|I^{*}\right|}}+b_{n}\right)\right)\right. \\
& \left.\geq \boldsymbol{Z}(\tilde{I})-\sqrt{2 \log \frac{e n}{|\tilde{I}|}}+\left(1-\frac{1}{3 \sqrt{\log _{2} \frac{n}{\left|I^{*}\right|}}}\right)\left(\sqrt{2 \log \frac{e n}{\left|I^{*}\right|}}+b_{n}\right)\right) \\
& \leq P_{H_{1}}\left(\max _{I \in K_{1} \backslash K_{\text {near }}}\left(\boldsymbol{Z}(I)-\sqrt{2 \log \frac{e n}{|I|}}\right)\right. \\
& \geq \boldsymbol{Z}(\tilde{I})-\left(\sqrt{2 \log \frac{e n}{|\tilde{I}|}}-\sqrt{2 \log \frac{e n}{\left|I^{*}\right|}}\right) \\
& \left.-\left(\frac{1}{3 \sqrt{\log \frac{n}{I^{*} \mid}}}+\frac{1}{\sqrt{\log n}}\right)\left(\sqrt{2 \log \frac{e n}{\left|I^{*}\right|}}\right)+\left(1-\frac{1}{3 \sqrt{\log _{2} \frac{n}{\left|I^{*}\right|}}}-\frac{1}{\sqrt{\log n}}\right) b_{n}\right)
\end{aligned}
$$

Since $\max _{I \in K_{1} \backslash K_{\text {near }}}\left(\boldsymbol{Z}(I)-\sqrt{2 \log \frac{e n}{|I|}}\right)=O_{p}(1)$ by Lemma $8, \boldsymbol{Z}(\tilde{I})=O_{p}(1)$, $\left(\frac{1}{3 \sqrt{\log _{2} \frac{n}{I^{*} \mid}}}+\frac{1}{\sqrt{\log n}}\right)\left(\sqrt{2 \log \frac{e n}{\left|I^{*}\right|}}\right)=O(1), \sqrt{2 \log \frac{e n}{|\tilde{I}|}}-\sqrt{2 \log \frac{e n}{\left|I^{*}\right|}}=o(1)$ by Lemma 9 and $\left(1-\frac{1}{3 \sqrt{\log _{2} \frac{n}{I^{*} \mid}}}-\frac{1}{\sqrt{\log n}}\right) b_{n} \rightarrow \infty$, then $P_{H_{1}}\left(\max _{I \in K_{1} \backslash K_{\text {near }}} \boldsymbol{X}(I) \geq\right.$ $\boldsymbol{X}(\tilde{I})) \rightarrow 0$ and we finish our proof.

\section{A.2 Proofs for Section 3}

\section{Proof of Proposition 3 ;}

Proof. Denote $\# \mathcal{I}_{a p p}^{\{2\}}(\ell)$ be the number of rectangles in $\mathcal{I}_{a p p}^{\{2\}}(\ell)$. A simple counting shows that when $\ell \leq\left\lfloor\log _{2} \frac{n^{2}}{\log n}\right\rfloor, \# I_{a p p}^{\{2\}}(\ell)=2 \ell^{3} 2^{\ell}$. Let's abuse the notation a bit: for $\left\lceil\log _{2} n^{2}\right\rceil \geq \ell>\left\lfloor\log _{2} \frac{n^{2}}{\log n}\right\rfloor$, let $I_{a p p}^{\{2\}}(\ell)=\left\{I \in I_{\text {small }}, n^{2} 2^{-\ell}<|I| \leq\right.$ $\left.2 n^{2} 2^{-\ell}\right\}$. Then simple counting again gives $\# I_{a p p}^{\{2\}}(\ell) \leq n^{2} \sum_{|I|=n^{2} 2^{-\ell}}^{2 n^{2} 2^{-\ell}}|I| \leq$ $2 n^{4} 2^{-\ell} \log n \leq 2 \log ^{3} n 2^{\ell} \leq 2 \ell^{3} 2^{\ell}$, where the the second to the last inequality comes from $\ell>\left\lfloor\log _{2} \frac{n^{2}}{\log n}\right\rfloor$, so $2^{2 l} \geq \frac{n^{4}}{\log ^{2} n}$ and last inequality comes from $\ell>\left\lfloor\log _{2} \frac{n^{2}}{\log n}\right\rfloor \geq \log n$. Thus for all $1 \leq \ell \leq\left\lceil\log _{2} n^{2}\right\rceil$, we have $\# I_{a p p}^{\{2\}}(\ell) \leq 2 \ell^{3} 2^{\ell}$. 
For $\kappa>2$, we obtain:

$$
\begin{aligned}
& P\left(\max _{I \in I_{a p p}^{\{2\}}}\left(\boldsymbol{Y}(I)-\sqrt{2 \log \frac{e n^{2}}{|I|}}\right)>\kappa\right) \\
\leq & \sum_{\ell=1}^{\left\lceil\log _{2} n^{2}\right\rceil} \# I_{a p p}^{\{2\}}(\ell) \max _{I \in I_{a p p}(\ell)} \exp \left(-\frac{1}{2}\left(\sqrt{2 \log \frac{e n^{2}}{|I|}}+\kappa\right)^{2}\right) \\
\leq & \sum_{\ell=1}^{\left\lceil\log _{2} n^{2}\right\rceil} 2 \ell^{3} 2^{\ell} 2^{-\ell} \exp \left(-\kappa \sqrt{\ell}-\kappa^{2} / 2\right) \\
= & \sum_{\ell=1}^{\left\lceil\log _{2} n^{2}\right\rceil} 2 \ell^{3} \exp \left(-\kappa \sqrt{\ell}-\kappa^{2} / 2\right) \\
\leq & C \exp \left(-\kappa^{2} / 2\right)
\end{aligned}
$$

for some constant $C>0$ not depending on $n$. Thus, we have

$$
\max _{I \in I_{a p p}^{\{2\}}}\left(\boldsymbol{Y}(I)-\sqrt{2 \log \frac{e n^{2}}{|I|}}\right)=O_{p}(1) .
$$

Analogously to Lemma 9, the following lemma shows that in the two-dimensional case, we can also approximate every rectangle well enough by $\mathcal{I}_{a p p}^{\{2\}}$.

Lemma 12. For each $I^{*}$ with $\left|I^{*}\right| \ll n^{2}$, there exists an $\tilde{I} \in \mathcal{I}_{a p p}^{\{2\}}$ such that $D\left(\tilde{I}, I^{*}\right) \leq \frac{6}{\sqrt{\log _{2} \frac{n^{2}}{\left|I^{*}\right|}}}$ and $\sqrt{2 \log \frac{e n^{2}}{|\tilde{I}|}}-\sqrt{2 \log \frac{e n^{2}}{\left|I^{*}\right|}}=o(1)$.

The proof of this lemma is very similar to Lemma 9, and thus is omitted. See also [23].

\section{Proof of Theorem 4;}

Proof. Denote $\boldsymbol{X}(I)=\boldsymbol{Y}(I)-\sqrt{2 \log \frac{e n^{2}}{|I|}}$ for rectangle $I$.

By the definition of $\gamma_{n}^{\{2\}}(\alpha)$,

$$
P_{H_{0}}(\hat{I} \neq \phi)=P_{H_{0}}\left(\max _{I \in \mathcal{I}_{a p p}} \boldsymbol{X}(I) \geq \gamma_{n}^{\{2\}}(\alpha)\right) \leq \alpha,
$$

which proves (2).

Now we turn to prove (3). Again, let $I^{*}$ denote the true rectangle with length $a^{*}$ and width $b^{*}$. Define

$$
K_{0}=\left\{I \in \mathcal{I}_{a p p}^{\{2\}}: I \cap I^{*}=\emptyset\right\}
$$

and

$$
K_{1}=\left\{I \in \mathcal{I}_{a p p}^{\{2\}}: I \cap I^{*} \neq \emptyset \text { and } D\left(I, I^{*}\right)>\delta_{n}\right\} .
$$


By Lemma 12, there exists an rectangle $\tilde{I} \in \mathcal{I}_{a p p}^{\{2\}}$, such that $D\left(\tilde{I}, I^{*}\right) \leq \frac{6}{\sqrt{\log _{2} \frac{n^{2}}{\left|I^{*}\right|}}}$ and $\sqrt{2 \log \frac{e n^{2}}{|\tilde{I}|}}-\sqrt{2 \log \frac{e n^{2}}{\left|I^{*}\right|}}=o(1)$. Then,

$$
\begin{aligned}
P_{H_{1}}\left(D\left(\hat{I}, I^{*}\right)>\delta_{n}\right) & \leq P_{H_{1}}\left(\max \left(\max _{I \in \mathcal{I}_{a p p}^{\{2\}}, D\left(I, I^{*}\right)>\delta_{n}} \boldsymbol{X}(I), \gamma_{n}^{\{2\}}(\alpha)\right) \geq \boldsymbol{X}(\tilde{I})\right) \\
& \leq P_{H_{1}}\left(\max _{I \in K_{0}} \boldsymbol{X}(I) \geq \boldsymbol{X}(\tilde{I})\right)+P_{H_{1}}\left(\max _{I \in K_{1}}(\boldsymbol{X}(I) \geq \boldsymbol{X}(\tilde{I}))+P_{H_{1}}\left(\gamma_{n}^{\{2\}}(\alpha) \geq \boldsymbol{X}(\tilde{I})\right) .\right.
\end{aligned}
$$

By Proposition 3, $\max _{I \in K_{0}} \boldsymbol{X}(I)=O_{p}(1)$ and $\gamma_{n}^{\{2\}}(\alpha)<\infty$ a.s.. Notice that under $H_{1}$, by Lemma12 and the same reasoning as in the proof of Theorem 2, we have $X(\tilde{I}) \stackrel{p}{\rightarrow} \infty$. Thus $P_{H_{1}}\left(\max _{I \in K_{0}} \boldsymbol{X}(I) \geq X(\tilde{I})\right) \rightarrow 0$ and $P_{H_{1}}\left(\gamma_{n}^{\{2\}}(\alpha) \geq\right.$ $\boldsymbol{X}(\tilde{I})) \rightarrow 0$.

Now consider the term $P_{H_{1}}\left(\max _{I \in K_{1}}(\boldsymbol{X}(I) \geq \boldsymbol{X}(\tilde{I}))\right.$. Denote

$$
K_{\text {near }}=\left\{I \in K_{1}: \frac{a^{*}}{\log n} \leq a \leq a^{*} \log n \text { and } \frac{b^{*}}{\log n} \leq b \leq b^{*} \log n\right\},
$$

then

$$
\begin{aligned}
P_{H_{1}}\left(\max _{I \in K_{1}} X(I) \geq X(\tilde{I})\right) & \leq \sum_{I \in K_{\text {near }}} P_{H_{1}}(\boldsymbol{X}(I) \geq \boldsymbol{X}(\tilde{I}))+P_{H_{1}}\left(\max _{I \in K_{1} \backslash K_{\text {near }}} \boldsymbol{X}(I) \geq \boldsymbol{X}(\tilde{I})\right) \\
& =: \quad(A)+(B)
\end{aligned}
$$

Consider part (A) first. Similar to the proof of Theorem 2, one can show that for all $I \in K_{\text {near }}$

$$
P_{H_{1}}(\boldsymbol{X}(I) \geq \boldsymbol{X}(\tilde{I})) \leq \log ^{-\eta} n
$$

for all $\eta>0$. Simple counting shows the cardinality of the set $K_{\text {near }}$ is $O\left(\log ^{4} n\right)$. Thus

$$
\sum_{I \in K_{\text {near }}} P_{H_{1}}\left(\boldsymbol{X}(I) \geq \boldsymbol{X}\left(I^{*}\right)\right) \rightarrow 0
$$


Consider part (B), in this case, $1-D\left(I, I^{*}\right) \leq 1 / \sqrt{\log n}$. Thus

$$
\begin{aligned}
& P_{H_{1}}\left(\max _{I \in K_{1} \backslash K_{\text {near }}} \boldsymbol{X}(I) \geq \boldsymbol{X}(\tilde{I})\right) \\
& =P_{H_{1}}\left(\max _{I \in K_{1} \backslash K_{\text {near }}}\left(\boldsymbol{Z}(I)+\left(1-D\left(I, I^{*}\right)\right)\left(\sqrt{2 \log \frac{e n^{2}}{\left|I^{*}\right|}}+b_{n}\right)-\sqrt{2 \log \frac{e n^{2}}{|I|}}\right)\right. \\
& \left.\left.\geq \boldsymbol{Z}(\tilde{I})+\left(1-D\left(\tilde{I}, I^{*}\right)\right)\left(\sqrt{2 \log \frac{e n^{2}}{\left|I^{*}\right|}}+b_{n}\right)-\sqrt{2 \log \frac{e n^{2}}{|\tilde{I}|}}\right)\right) \\
& \leq P_{H_{1}}\left(\max _{I \in K_{1} \backslash K_{\text {near }}}\left(\boldsymbol{Z}(I)-\sqrt{2 \log \frac{e n^{2}}{|I|}}+\frac{1}{\sqrt{\log n}}\left(\sqrt{2 \log \frac{e n^{2}}{\left|I^{*}\right|}}+b_{n}\right)\right)\right. \\
& \left.\geq \boldsymbol{Z}(\tilde{I})-\sqrt{2 \log \frac{e n^{2}}{|\tilde{I}|}}+\left(1-\frac{6}{\sqrt{\log _{2} \frac{n^{2}}{\left|I^{*}\right|}}}\right)\left(\sqrt{2 \log \frac{e n^{2}}{\left|I^{*}\right|}}+b_{n}\right)\right) \\
& \leq P_{H_{1}}\left(\max _{I \in K_{1} \backslash K_{\text {near }}}\left(\boldsymbol{Z}(I)-\sqrt{2 \log \frac{e n^{2}}{|I|}}\right) \geq \boldsymbol{Z}(\tilde{I})-\left(\sqrt{2 \log \frac{e n^{2}}{|\tilde{I}|}}-\sqrt{2 \log \frac{e n^{2}}{\left|I^{*}\right|}}\right)\right. \\
& \left.-\left(\frac{6}{\sqrt{\log \frac{n^{2}}{\left|I^{*}\right|}}}+\frac{1}{\sqrt{\log n}}\right)\left(\sqrt{2 \log \frac{e n^{2}}{\left|I^{*}\right|}}\right)+\left(1-\frac{6}{\sqrt{\log _{2} \frac{n^{2}}{I^{*} \mid}}}-\frac{1}{\sqrt{\log n}}\right) b_{n}\right)
\end{aligned}
$$

Since $\max _{I \in K_{1} \backslash K_{\text {near }}}\left(\boldsymbol{Z}(I)-\sqrt{2 \log \frac{e n^{2}}{|I|}}\right)=O_{p}(1)$ by Proposition $3, \boldsymbol{Z}(\tilde{I})=$ $O_{p}(1),\left(\frac{6}{\sqrt{\log _{2} \frac{n^{2}}{I^{*}}}}+\frac{1}{\sqrt{\log n}}\right)\left(\sqrt{2 \log \frac{e n^{2}}{\left|I^{*}\right|}}\right)=O(1),\left(\sqrt{2 \log \frac{e n^{2}}{|\tilde{I}|}}-\sqrt{2 \log \frac{e n^{2}}{\left|I^{*}\right|}}\right)=$ $o(1)$ by Lemma 12, and $\left(1-\frac{6}{\sqrt{\log _{2} \frac{n^{2}}{\left|I^{*}\right|}}}-\frac{1}{\sqrt{\log n}}\right) b_{n} \rightarrow \infty$, then $P_{H_{1}}\left(\max _{I \in K_{1} \backslash K_{\text {near }}} \boldsymbol{X}(I) \geq\right.$ $\boldsymbol{X}(\tilde{I})) \rightarrow 0$.

\section{A.3 Proof for Section 4}

We need the following lemma in the proof of Theorem 6 ,

Lemma 13. Denote $L=\max _{I \in I_{a p p}}\left(\boldsymbol{Y}(I)-\sqrt{2 \log \frac{e n}{\mid T}}\right)$. Let $Z$ be a standard normal random variable, not necessarily independent with $L$, then for all $\kappa>4$, there exists a constant $C>0$ not depending on $n$ and $\kappa$ such that

$$
P(L+Z>\kappa) \leq C \exp \left(-\kappa^{2} / 8\right)
$$

Proof. Similar to the proof of Proposition 3, we know that when $\kappa>2, P(L>$ $\kappa) \leq C^{\prime} \exp \left(-\kappa^{2} / 2\right)$ for some $C^{\prime}>0$ not depending on $n$ and $\kappa$. Thus,

$$
\begin{aligned}
P(L+Z>\kappa) & \leq P(L>\kappa / 2)+P(Z>\kappa / 2) \\
& \leq C^{\prime} \exp \left(-\kappa^{2} / 8\right)+2 \exp \left(-\kappa^{2} / 8\right) \\
& \leq C \exp \left(-\kappa^{2} / 8\right) .
\end{aligned}
$$




\section{Proof of Theorem 6:}

Proof. As before, denote $\boldsymbol{X}(I)=\boldsymbol{Y}(I)-\sqrt{2 \log \frac{e n}{|T|}}$ for interval $I$.

When there exists no signal, by the definition of $M_{n}$,

$$
P_{H_{0}}(I \neq \emptyset)=P_{H_{0}}\left(\max _{I \in \mathcal{I}_{a p p}} \boldsymbol{X}(I)>\gamma_{n}\right) \leq \alpha,
$$

which proves (7).

Now we turn to prove (9). It is enough to show that with probability approaching 1 , we will not stop before the $K t h$ iteration and for each of the first $K$ iterations, we can correctly identify one of the true signals with precision $\delta_{n}$.

Recall the true signals $I^{*}=\left\{I_{\tilde{I}_{j}^{*}}^{*} I_{2}^{*}, \ldots, I_{K}^{*}\right\}$. By Lemma 9 for each $j=$ $1, \ldots, K$, there exists an interval $\tilde{I}_{j}$, such that $D\left(\tilde{I}_{j}, I_{j}^{*}\right) \leq \frac{1}{\sqrt[3]{\log _{2} \frac{n}{\left|I_{j}^{*}\right|}}}$ and $\sqrt{2 \log \frac{e n}{\left|\tilde{I}_{j}\right|}}-\sqrt{2 \log \frac{e n}{\left|I_{j}^{*}\right|}}=o(1)$.

Consider the event

$$
E_{1}=\left\{\max \left(\max _{I \in \mathcal{I}_{a p p}^{1}, \min _{j=1}^{K} D\left(I, I_{j}^{*}\right)>\delta_{n}} \boldsymbol{X}(I), \gamma_{n}(\alpha)\right)<\min _{j=1}^{K} \boldsymbol{X}\left(\tilde{I}_{j}\right)\right\}:=\{L H S<R H S\} .
$$

If $E_{1}$ holds, then we can be sure that the interval $\hat{I}_{1}$ identified by the first iteration satisfies $D\left(\hat{I}_{1}, I_{j_{1}}^{*}\right)<\delta_{n}$ for some $j_{1}$ in 1 to $K$ and $\hat{I}_{1} \cap I_{j}^{*}=\emptyset$ for all $j \neq j_{1}$ by the assumption of $d_{\min }$. After the first iteration, consider the event

$$
E_{2}=\left\{\max \left(\underset{I \in \mathcal{I}_{a p p}^{2}, \min _{j=1, j \neq j_{1}}^{K} D\left(I, I_{j}^{*}\right)>\delta_{n}}{\max } \boldsymbol{X}(I), \gamma_{n}(\alpha)\right)<\min _{j=1, j \neq j_{1}}^{K} \boldsymbol{X}\left(\tilde{I}_{j}\right)\right\}:=\{L H S<R H S\},
$$

the LHS of $E_{2}$ is non-increasing while the RHS of $E_{2}$ is non-decreasing compared to those of $E_{1}$. Thus, if $E_{1}$ holds, $E_{2}$ must hold, and we can be sure that the interval $\hat{I}_{2}$ identified by the second iteration satisfies $D\left(\hat{I}_{2}, I_{j_{2}}^{*}\right) \leq \delta_{n}$ for some $j_{2}$ from 1 to $K, j_{2} \neq j_{1}$ and $\hat{I}_{2} \cap I_{j}^{*}=\emptyset$ for all $j \neq j_{2}$. If this procedure can be repeated for $K$ times, then we can identify all $K$ signals with precision $\delta_{n}$. Thus,

$$
\begin{aligned}
& P_{H_{1}}\left(\underset{j=1}{K} \underset{i=1}{\hat{K}} \min _{i=1}^{\hat{K}} D\left(\hat{I}_{i}, I_{j}^{*}\right)>\delta_{n}\right) \\
\leq & P_{H_{1}}\left(E_{1}^{C}\right) \\
= & P_{H_{1}}\left(\max \left(\underset{I \in \mathcal{I}_{a p p}^{1}, \min _{j=1}^{K} D\left(I, I_{j}^{*}\right)>\delta_{n}}{X} \boldsymbol{X}(I), \gamma_{n}(\alpha)\right) \geq \min _{j=1}^{K} \boldsymbol{X}\left(\tilde{I}_{j}\right)\right)
\end{aligned}
$$

Define

$$
K_{0}=\left\{I \in \mathcal{I}_{\text {app }}: I \cap I_{j}^{*}=\emptyset \text { for all } j=1, \ldots, K\right\}
$$

and

$$
K_{1}=\left\{I \in \mathcal{I}_{\text {app }}: I \cap I_{j}^{*} \neq \emptyset \text { for some } j=1, \ldots, K \text { and } \underset{j=1}{K} D\left(I, I_{j}^{*}\right)>\delta_{n}\right\} .
$$


Then

$$
\begin{aligned}
& P_{H_{1}}\left(\max \left(\underset{I \in \mathcal{I}_{a p p}^{1}, \min _{j=1}^{K} D\left(I, I_{j}^{*}\right)>\delta_{n}}{\max _{\max ^{\prime}}} \boldsymbol{X}(I), \gamma_{n}(\alpha)\right) \geq \min _{j=1}^{K} \boldsymbol{X}\left(\tilde{I}_{j}\right)\right) \\
\leq & P_{H_{1}}\left(\max _{I \in K_{0}} \boldsymbol{X}(I) \geq \underset{j=1}{K} \boldsymbol{X}\left(\tilde{I}_{j}\right)\right)+P_{H_{1}}\left(\max _{I \in K_{1}} \boldsymbol{X}(I) \geq \underset{j=1}{K} \boldsymbol{X}\left(\tilde{I}_{j}\right)\right)+P_{H_{1}}\left(\gamma_{n}(\alpha) \geq \underset{j=1}{K} \boldsymbol{X}\left(\tilde{I}_{j}\right)\right)
\end{aligned}
$$

By Lemma 8, $\max _{I \in K_{0}} \boldsymbol{X}(I) \stackrel{d}{\leq} L<\infty$ a.s. and $\gamma_{n}<\infty$ a.s.. Notice that under $H_{1}$ and our assumption $b_{n, j} \gg \sqrt{\log \log n}$, each $X\left(\tilde{I}_{j}\right)$ is Gaussian distributed with mean greater than

$$
\left(\sqrt{2 \log \frac{e n}{\left|I_{j}^{*}\right|}}+b_{n}\right)\left(1-\frac{1}{3 \sqrt{\log \frac{n}{\left|I_{j}^{*}\right|}}}\right)-\sqrt{2 \log \frac{e n}{\left|\tilde{I}_{j}\right|}} \gg \sqrt{\log \log n}
$$

and variance 1 . Then similar to the proof of Theorem 2 for each $j=1, \ldots, K$, and $\eta>0$, we have

$$
P_{H_{1}}\left(\max _{I \in K_{0}} \boldsymbol{X}(I) \geq \boldsymbol{X}\left(\tilde{I}_{j}\right)\right) \leq \log ^{-\eta} n
$$

and

$$
P_{H_{1}}\left(\gamma_{n}(\alpha) \geq \boldsymbol{X}\left(\tilde{I}_{j}\right)\right) \leq \log ^{-\eta} n .
$$

As a result, under the assumption that $K=\log ^{p} n$ for some $p>0, P_{H_{1}}\left(\max _{I \in K_{0}} \boldsymbol{X}(I) \geq\right.$ $\left.\min _{j=1}^{K} \boldsymbol{X}\left(\tilde{I}_{j}\right)\right) \leq K P_{H_{1}}\left(\max _{I \in K_{0}} \boldsymbol{X}(I) \geq \boldsymbol{X}\left(\tilde{I}_{j}\right)\right) \rightarrow 0$ and $P_{H_{1}}\left(\gamma_{n}(\alpha) \geq\right.$ $\left.\min _{j=1}^{K} \boldsymbol{X}\left(\tilde{I}_{j}\right)\right) \leq K P_{H_{1}}\left(\gamma_{n}(\alpha) \geq \boldsymbol{X}\left(\tilde{I}_{j}\right)\right) \rightarrow 0$.

We only need to show that $P_{H_{1}}\left(\max _{I \in K_{1}} \boldsymbol{X}(I) \geq \min _{j=1}^{K} \boldsymbol{X}\left(\tilde{I}_{j}\right)\right) \rightarrow 0$. Denote

$$
K_{\text {near }}=\left\{I \in K_{1}: \min _{j=1}^{K} D\left(I, I_{j}^{*}\right)<1-1 / \sqrt{\log n}\right\},
$$

then

$$
\begin{aligned}
P_{H_{1}}\left(\max _{I \in K_{1}} \boldsymbol{X}(I) \geq \min _{j=1}^{K} \boldsymbol{X}\left(\tilde{I}_{j}\right)\right) & \leq \sum_{j=1}^{K} \sum_{I \in K_{\text {near }}} P_{H_{1}}\left(\boldsymbol{X}(I) \geq \boldsymbol{X}\left(\tilde{I}_{j}\right)\right)+\sum_{j=1}^{K} P_{H_{1}}\left(\max _{I \in K_{1} \backslash K_{\text {near }}} \boldsymbol{X}(I) \geq \boldsymbol{X}(\tilde{I})\right) \\
=: & (A)+(B)
\end{aligned}
$$

For part (A), as in the proof of Theorem 2, for each $j=1, \ldots, K, P_{H_{1}}(\boldsymbol{X}(I) \geq$ $\left.\boldsymbol{X}\left(\tilde{I}_{j}\right)\right) \leq \log ^{-\eta} n$ for all $\eta>0$. Note that if any signal $I$ intersects with more than one element in $I^{*}$, then we must have $\min _{j=1}^{K} D\left(I, I_{j}^{*}\right) \geq 1-1 / \sqrt{\log n}$ by our assumption of $d_{\text {min }}$, and thus such $I \notin K_{\text {near }}$. Thus, the cardinality of the set $K_{\text {near }}$ is $O\left(\log ^{2} n K\right)=O\left(\log ^{p+2} n\right)$. As a result,

$$
\sum_{j=1}^{K} \sum_{I \in K_{\text {near }}} P_{H_{1}}\left(\boldsymbol{X}(I) \geq \boldsymbol{X}\left(I_{j}^{*}\right)\right) \rightarrow 0
$$


For part $(\mathrm{B})$, in this situation $1-D\left(I, I_{j}^{*}\right) \leq 1 / \sqrt{\log n}$ for all $j=1, \ldots, K$. Thus

$$
\begin{aligned}
& P_{H_{1}}\left(\max _{I \in K_{1} \backslash K_{n e a r}} \boldsymbol{X}(I) \geq \boldsymbol{X}\left(\tilde{I}_{j}\right)\right) \\
& =P_{H_{1}}\left(\max _{I \in K_{1} \backslash K_{n e a r}}\left(\boldsymbol{Z}(I)+\left(1-D\left(I, I_{j}^{*}\right)\right)\left(\sqrt{2 \log \frac{e n}{\left|I_{j}^{*}\right|}}+b_{n, j}\right)-\sqrt{2 \log \frac{e n}{|I|}}\right)\right. \\
& \left.\left.\geq \boldsymbol{Z}\left(\tilde{I}_{j}\right)+\left(1-D\left(\tilde{I}_{j}, I_{j}^{*}\right)\right)\left(\sqrt{2 \log \frac{e n}{\left|I_{j}^{*}\right|}}+b_{n, j}\right)-\sqrt{2 \log \frac{e n}{\left|\tilde{I}_{j}\right|}}\right)\right) \\
& \leq P_{H_{1}}\left(\max _{I \in K_{1} \backslash K_{\text {near }}}\left(\boldsymbol{Z}(I)-\sqrt{2 \log \frac{e n}{|I|}}+\frac{1}{\sqrt{\log n}}\left(\sqrt{2 \log \frac{e n}{\left|I_{j}^{*}\right|}}+b_{n, j}\right)\right)\right. \\
& \left.\geq \boldsymbol{Z}\left(\tilde{I}_{j}\right)-\sqrt{2 \log \frac{e n}{\left|\tilde{I}_{j}\right|}}+\left(1-\frac{1}{3 \sqrt{\log \frac{n}{\left|I_{j}^{*}\right|}}}\right)\left(\sqrt{2 \log \frac{e n}{\left|I_{j}^{*}\right|}}+b_{n, j}\right)\right) \\
& \leq P_{H_{1}}\left(\max _{I \in K_{1} \backslash K_{\text {near }}}\left(\boldsymbol{Z}(I)-\sqrt{2 \log \frac{e n}{|I|}}\right)\right. \\
& \geq \boldsymbol{Z}\left(\tilde{I}_{j}\right)-\left(\sqrt{2 \log \frac{e n}{\left|\tilde{I}_{j}\right|}}-\sqrt{2 \log \frac{e n}{\left|I_{j}^{*}\right|}}\right) \\
& -\left(\frac{1}{3 \sqrt{\log \frac{n}{\left|I_{j}^{*}\right|}}}+\frac{1}{\sqrt{\log n}}\right)\left(\sqrt{2 \log \frac{e n}{\left|I_{j}^{*}\right|}}\right)+\left(1-\frac{1}{3 \sqrt{\log \frac{n}{I_{j}^{*} \mid}}}-\frac{1}{\sqrt{\log n}}\right) b_{n, j}
\end{aligned}
$$

By Lemma 13. $P_{H_{1}}\left(\max _{I \in K_{1} \backslash K_{n e a r}}\left(\boldsymbol{Z}(I)-\sqrt{2 \log \frac{e n}{|I|}}\right)-Z\left(\tilde{I}_{j}\right)>\kappa\right) \leq$ $C \exp \left(-\kappa^{2} / 8\right)$. Notice that $\left(\frac{1}{3 \sqrt{\log \frac{n}{\left|I_{j}^{*}\right|}}}+\frac{1}{\sqrt{\log n}}\right)\left(\sqrt{2 \log \frac{e n}{\left|I_{j}^{*}\right|}}\right)=O(1)$ and $(1-$ $\left.\frac{1}{3 \sqrt{\log \frac{n}{\left|I_{j}^{*}\right|}}}-\frac{1}{\sqrt{\log n}}\right) b_{n, j} \gg \sqrt{\log \log n}$, thus $P_{H_{1}}\left(\max _{\hat{I} \in K_{1} \backslash K_{n e a r}} \boldsymbol{X}(I) \geq \boldsymbol{X}\left(\tilde{I}_{j}\right)\right) \leq$ $\log ^{-\eta} n$ for all $\eta>0$. So $\sum_{j=1}^{K} P_{H_{1}}\left(\max _{I \in K_{1} \backslash K_{n e a r}} \boldsymbol{X}(I) \geq \boldsymbol{X}\left(\tilde{I}_{j}\right)\right) \rightarrow 0$. As a result, $P_{H_{1}}\left(\max _{\hat{I} \in K_{1}}\left(\boldsymbol{X}(I) \geq \min _{j=1}^{K} \boldsymbol{X}\left(\tilde{I}_{j}\right)\right) \rightarrow 0\right.$ and we finish our proof for (9).

Note that for each iteration, we may only remove an interval very close to $I_{j}^{*}$ for some $j=1, \ldots, K$. So after $K$ iteration, there may still exist intervals $I \in$ $\mathcal{I}_{\text {app }}^{K}$ such that $I \cap I_{j}^{*} \neq \emptyset$ for some $j$, denote these intervals by $\mathcal{I}_{\text {left }}$. By similar argument as above, we can show that $P\left(\max _{I \in \mathcal{I}_{l e f t}} X(I)>\gamma_{n}(\alpha)\right) \rightarrow 0$. Now consider all intervals in $\mathcal{I}_{a p p}^{K} \backslash \mathcal{I}_{\text {left }}$, By the definition of $\gamma_{n}(\alpha)$ and noticing the fact that $\gamma_{n}(\alpha)$ is non-decreasing in $n$, we can conclude that the number of the false intervals our procedure identifies is controlled by a geometric distribution with parameter $\alpha$. Thus $E \hat{K} \leq K+\frac{\alpha}{1-\alpha}+o(1)$ and (8) follows by letting $C(\alpha)=\frac{\alpha}{1-\alpha}$.

\section{Proof of Theorem 7;}

Proof. Assuming without loss of generality that $\frac{n}{\left|I_{1}^{*}\right|}$ is an integer. Assume first that the signals can only start and end in a grid given by $\left\{i\left|I_{1}^{*}\right|+1, \ldots(i+1)\left|I_{1}^{*}\right|\right\}$ 
for $i=1, \ldots, \frac{n}{\left|I_{1}^{*}\right|}$. According to [16], it is enough to show that Theorem 7 holds under this assumption. Let $R_{i}=\sum_{j=1}^{\left|I_{1}^{*}\right|} X_{i\left|I_{1}^{*}\right|+j} / \sqrt{\left|I_{1}^{*}\right|}=: r_{i}+Z_{i}^{\prime}$ for $i=0, \ldots, n^{\prime}-1$, where $n^{\prime}=\frac{n}{\left|I_{1}^{*}\right|}$. Then $Z_{i}^{\prime} \stackrel{i i d}{\sim} N(0,1)$, and $r_{i}=0$ for all but $K$ locations, while at these locations, $r_{i} \leq \sqrt{2 \log \frac{e n}{\left|I_{1}^{*}\right|}}=\sqrt{2 \log e n^{\prime}}$. Since $\log K=b \log \log n=o(\log n)$, by Lemma 1 in [16, no identification procedure can be consistent under this model.

\section{References}

\section{References}

[1] E. Arias-Castro, E. J. Candès, and A. Durand. Detection of an anomalous cluster in a network. The Annals of Statistics, pages 278-304, 2011. 1, 2 , 6.1

[2] E. Arias-Castro, D. L. Donoho, and X. Huo. Near-optimal detection of geometric objects by fast multiscale methods. Information Theory, IEEE Transactions on, 51(7):2402-2425, 2005. 1, 1, 2

[3] L. D. Brown, T. T. Cai, and H. H. Zhou. Robust nonparametric estimation via wavelet median regression. The Annals of Statistics, pages 2055-2084, 2008. 6.2

[4] V.-E. Brunel. Convex set detection. arXiv preprint arXiv:1404.6224, 2014. 6.3

[5] H. P. Chan. Detection of spatial clustering with average likelihood ratio test statistics. The Annals of Statistics, 37(6B):3985-4010, 2009. 1

[6] H. P. Chan and G. Walther. Detection with the scan and the average likelihood ratio. Statistica Sinica, 23, 2013. 1, 2, 2, 3, 6.3

[7] A. Delaigle and P. Hall. Higher criticism in the context of unknown distribution, non-independence and classification. Perspectives in Math-ematical Sciences I: Probability and Statistics, pages 109-138, 2009. 1]

[8] D. Donoho and J. Jin. Higher criticism for detecting sparse heterogeneous mixtures. Annals of Statistics, pages 962-994, 2004. 1

[9] D. Donoho and J. Jin. Higher criticism for large-scale inference, especially for rare and weak effects. Statistical Science, 30(1):1-25, 2015. 1

[10] L. Dumbgen and V. G. Spokoiny. Multiscale testing of qualitative hypotheses. Annals of Statistics, pages 124-152, 2001. 1, 6.3

[11] L. Dümbgen and G. Walther. Multiscale inference about a density. The Annals of Statistics, pages 1758-1785, 2008. 1 
[12] K. Frick, A. Munk, and H. Sieling. Multiscale change point inference. Journal of the Royal Statistical Society: Series B (Statistical Methodology), 76(3):495-580, 2014. 6.1, 6.3

[13] R. E. Gangnon and M. K. Clayton. A weighted average likelihood ratio test for spatial clustering of disease. Statistics in Medicine, 20(19):2977-2987, 2001. 1

[14] C. Genovese, J. Jin, and L. Wasserman. Revisiting marginal regression. arXiv preprint arXiv:0911.4080, 2009. 4

[15] J. Glaz and N. Balakrishnan. Scan statistics and applications. Springer Science \& Business Media, 2012. 1

[16] X. J. Jeng, T. T. Cai, and H. Li. Optimal sparse segment identification with application in copy number variation analysis. Journal of the American Statistical Association, 105(491):1156-1166, 2010. (document), 1, 2, 2, 4. 5. 6.3

[17] D. B. Neill. An empirical comparison of spatial scan statistics for outbreak detection. International Journal of Health Geographics, 8(1):1, 2009. 1

[18] D. B. Neill, A. W. Moore, F. Pereira, and T. M. Mitchell. Detecting significant multidimensional spatial clusters. In Advances in Neural Information Processing Systems, pages 969-976, 2004. 1, 2

[19] C. Rivera and G. Walther. Optimal detection of a jump in the intensity of a poisson process or in a density with likelihood ratio statistics. Scandinavian Journal of Statistics, 40(4):752-769, 2013. 1, 2, 2

[20] K. Rufibach and G. Walther. The block criterion for multiscale inference about a density, with applications to other multiscale problems. Journal of Computational and Graphical Statistics, 19(1):175-190, 2010. 1]

[21] B. E. Stranger, M. S. Forrest, M. Dunning, C. E. Ingle, C. Beazley, N. Thorne, R. Redon, C. P. Bird, A. de Grassi, C. Lee, et al. Relative impact of nucleotide and copy number variation on gene expression phenotypes. Science, 315(5813):848-853, 2007. 1

[22] T. Tony Cai, X. Jessie Jeng, and H. Li. Robust detection and identification of sparse segments in ultrahigh dimensional data analysis. Journal of the Royal Statistical Society: Series B (Statistical Methodology), 74(5):773-797, 2012. 6.2

[23] G. Walther. Optimal and fast detection of spatial clusters with scan statistics. The Annals of Statistics, 38(2):1010-1033, 2010. 10 2, $3,6.3$ 